\title{
Glycosyl flavones from Humulus japonicus suppress MMP-1 production via decreasing oxidative stress in UVB irradiated human dermal fibroblasts
}

\author{
Eui Jeong Nam ${ }^{1}$, Gyhye Yoo ${ }^{2}$, Joo Young Lee ${ }^{3}$, Myungsuk Kim², Changho Jhin ${ }^{2}$, Yang-Ju Son ${ }^{2}$, Sun Young Kim², \\ Sang Hoon Jung ${ }^{3} \mathcal{E}$ Chu Won $\mathrm{Nho}^{2, *}$ \\ ${ }^{1}$ New Product Development Team, COSMAXNBT, Seoul 06132, ${ }^{2}$ Smart Farm Research Center, Korea Institute of Science and Technology \\ (KIST) Gangneung Institute, Gangneung 25451, ${ }^{3}$ Natural Products Research Center, Korea Institute of Science and Technology (KIST) \\ Gangneung Institute, Gangneung 25451, Korea
}

Exposure to Ultraviolet (UV) light induces photoaging of skin, leading to wrinkles and sunburn. The perennial herb Humulus japonicus, widely distributed in Asia, is known to have antiinflammatory, antimicrobial, and antioxidant effects. However, the physiological activities of isolated compounds from $\boldsymbol{H}$. japonicus have rarely been investigated. This study focused on the isolation of active compounds from $H$. japonicus and the evaluation of their effects on photoaging in UVB-irradiated human fibroblast (Hs68) cells. When the extract and four fractions of $\boldsymbol{H}$. japonicus were treated respectively in UVB-irradiated Hs68 cells to investigate anti-photoaging effects, the ethyl acetate (EtOAc) fraction showed the strongest inhibitory effect on MMP1 secretion. From EtOAc fraction, we isolated luteolin-8-C-glucoside (1), apigenin-8-C-glucoside (2), and luteolin-7-O-glucoside (3). These compounds suppressed UVB-induced MMP-1 production by inhibiting the phosphorylation of the mitogen-activated protein kinases (MAPKs) and activator protein-1 (AP-1). When the antioxidant activity of the compounds were estimated by conducting western blot, calculating the bond dissociation energies of the $\mathrm{O}-\mathrm{H}$ bond (BDE) at different grade, and measuring radical scavenging activity, we found luteolin-8-C-glucoside (1) showed the strongest activity on the suppression of UVB-induced photoaging. These results demonstrate the inhibitory effect of three flavone glycosides derived from $\boldsymbol{H}$. japonicus on MMP-1 production, MAPK and AP-1 signaling, and oxidative stress; this could prove useful in suppressing UVB induced photoaging. [BMB Reports 2020; 53(7): 379-384]

*Corresponding author. Tel: +82-33-650-3420; Fax: +82-33-6503429; E-mail: cwnho@kist.re.kr

https://doi.org/10.5483/BMBRep.2020.53.7.253

Received 22 October 2019, Revised 11 November 2019, Accepted 7 January 2020

Keywords: Antioxidant, Flavone glycosides, Humulus japonicus, MMP-1, Photoaging

\section{INTRODUCTION}

Aging is a natural process resulting in the degeneration of the organs but premature aging can be induced by external factors such as diets and life styles (1). Photoaging of the skin is one of the symptoms of premature aging characterized by dryness, wrinkles, laxity, and uneven pigmentation $(2,3)$. UV irradiation through sunlight is a predominant factor inducing photoaging and has harmful effects including immune suppression and oxidative stress. Cumulative damage by chronic exposure to sunlight leads to photoaging of the skin, and ultimately skin cancer. The UV portion of sunlight is divided into UVA (320-400 nm), UVB (280-320 nm), and UVC (100-280 nm). Approximately $90-99 \%$ of the solar ultraviolet radiation (UVR) energy that reaches the earth's surface is UVA, whereas only $1-10 \%$ is UVB (4). However, UVB, which has a short wavelength and high energy, can penetrate the upper layers of the epidermis and cause severe damage to the skin, such as impairment of DNA synthesis and repair system, inflammation, production of reactive oxygen species (ROS), and alternation of signaling transduction (3). Therefore, the suppression of UVB effects is critical to inhibition of photoaging of the skin.

The dermis is the layer of skin beneath the epidermis and functions as a skin connective tissue. The extracellular matrix (ECM) in the dermis is primarily composed of type I collagen, which is an essential building block of human skin that is in charge of its strength and resilience (1). However, it has been reported that when the skin is exposed to UV radiation, collagen degeneration is increased, accompanied by the aberrant accumulation of elastin fibers and glycosaminoglycans (3). Collagen damage results from an increase of matrix metalloproteinases (MMPs) stimulated by ROS (5). MMPs are a family of enzymes and have the capacity to degrade components of the ECM. Among the different types of MMPs, MMP-1 is a major collagenolytic enzyme in response to UV exposure and responsible for degradation of the ECM. UV-induced ROS production activates MAPK signaling pathway including JNK, ERK, and p38 pathways that play an important role in trans-

ISSN: 1976-670X (electronic edition)

Copyright (c) 2020 by the The Korean Society for Biochemistry and Molecular Biology

(c) This is an open-access article distributed under the terms of the Creative Commons Attribution Non-Commercial License (http://creativecommons.org/licenses/by-nc/4.0) which permits unrestricted non-commercial use, distribution, and reproduction in any medium, provided the original work is properly cited. 
mitting environmental cues through cell surface receptors and induce the phosphorylation of c-Fos, which binding with c-Jun forms the activator protein-1 (AP-1) complex $(1,6)$. Because the AP-1 complex is a well-known transcription factor that induces MMP-1 expressions, it can be surmised that the suppression of MMP-1 elevated by MAPK and AP-1 signaling is a potential strategy in anti-photoaging.

Humulus japonicus Siebold \& Zucc, one of the family Cannabaceae, is a perennial herb that grows as a weed in Korea, China, and Japan (7). Traditionally, this plant has been used as a medicine for pulmonary tuberculosis, hypertension, leprosy, and venomous wounds, and as an ingredient of tea in China and Korea. $H$. japonicus has been reported its various medicinal activities such as anti-inflammatory (8) antitumor (9) and antioxidant activities (10). Its bioactive constituents such as terpenes (11), phenols (12), and flavonoids (7) have been isolated. Although $H$. japonicus extract has been investigated for its various bioactivities, the physiological activities of its isolated compounds have rarely been studied. In this study, we isolated three important bioactive compounds from its ethyl acetate (EtOAC) fraction based on their activities, and demonstrated the potential of complete $H$. japonicus extract and its compounds in anti-photoaging in UVB-irradiated human fibroblast (Hs68).

\section{RESULTS}

Inhibitory effects of $\boldsymbol{H}$. japonicus extract and EtOAc fraction on UVB-induced MMP-1 secretions in Hs68 cells

Before studying the anti-photoaging effects of $\mathrm{H}$. japonicus, we examined the cytotoxicity of $H$. japonicus extract (HJE) and $H$. japonicus fractions (HJF). The results of MTT assay showed that $\mathrm{HJE}, \mathrm{EtOAc}$, and $\mathrm{BuOH}$ fractions had no cytotoxicity at any concentration (Fig. $1 \mathrm{~A}$ and B). However, $\mathrm{CH}_{2} \mathrm{Cl}_{2}$ and $\mathrm{Hex}$ fractions exhibited a cytotoxicity at $6.25 \mu \mathrm{g} / \mathrm{ml}$ and at 25 $\mu \mathrm{g} / \mathrm{ml}$, respectively (Fig. 1B). Based on these data, when these fractions were applied for UVB-exposed fibroblasts, their concentration was $6.25 \mu \mathrm{g} / \mathrm{ml}$ or less.

To examine whether HJE inhibited MMP-1 production, we measured the concentration of MMP-1 secreted into the media in UVB-irradiated Hs68 cells. Epigallocatechin gallate (EGCG) was used as a positive control because it has been well known to inhibit MMP-1 production in human dermal fibroblasts (13). HJE significantly decreased MMP-1 secretion in a dose dependent manner (Fig. 1C). When fractions were treated at $20 \mu \mathrm{g} / \mathrm{ml}$ concentrations, EtOAc, $\mathrm{BtOH}$ and $\mathrm{CH}_{2} \mathrm{Cl}_{2}$ fractions showed strong inhibition of MMP-1 secretion (Fig. 1D).

\section{Suppressive effects of three flavone glycosides from the EtOAc fraction of $\boldsymbol{H}$. japonicus on MMP-1 secretion, and MAPKs and AP-1 activation in UVB-induced $\mathrm{Hs68}$ cells}

Considering severe toxicity of $\mathrm{CH}_{2} \mathrm{Cl}_{2}$ fraction and the effect of HJFs on MMP-1, we selected the EtOAc fraction as the most
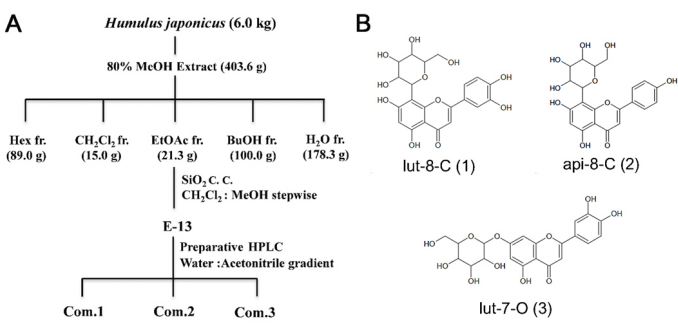

C

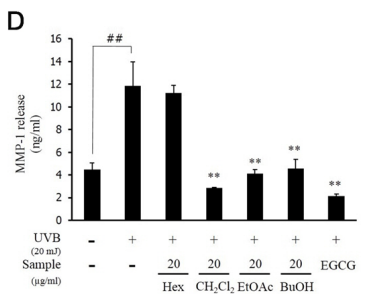

Fig. 1. Effects of HJE and HJF on cell viability and MMP-1 expression in UVB-induced Hs68 cells. Cell viability was examined with various concentration of $\mathrm{HJE}(\mathrm{A})$ and $\mathrm{HJF}(\mathrm{B})$ : $\mathrm{Hex}, \mathrm{CH}_{2} \mathrm{Cl}_{2}$, EtOAc, or $\mathrm{BuOH}$, with UVB $\left(20 \mathrm{~mJ} / \mathrm{cm}^{2}\right)$ in $\mathrm{Hs} 68$ cells. Quantified protein level of MMP-1 in cells pretreated with the indicated concentration of $\mathrm{HJE}$ (C) and $\mathrm{HJF}$ (D): $\mathrm{Hex}, \mathrm{CH}_{2} \mathrm{Cl}_{2}, \mathrm{EtOAc}$, or $\mathrm{BuOH}$, for $1 \mathrm{~h}$ and irradiated with UVB $\left(20 \mathrm{~mJ} / \mathrm{cm}^{2}\right)$. EGCG was used as a positive control. Results are expressed as mean \pm SD of triplicate experiments $\left({ }^{*} \mathrm{P}<0.05,{ }^{\# \#} \mathrm{P}<0.01,{ }^{* *} \mathrm{P}<0.01\right.$, $* * * * \mathrm{P}<0.0001)$

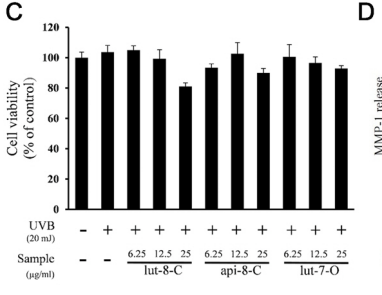

D

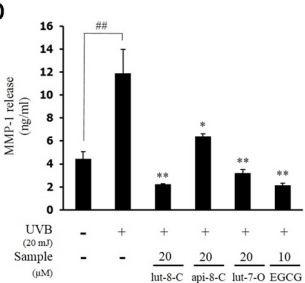

Fig. 2. Effects three flavone glycosides from EtOAc fraction on cell viability and MMP-1 expression in UVB-induced Hs68 cells. (A) Active guided fractionation of $\mathrm{HJE}$ (B) Chemical structure of isolated compound (1), (2), and (3) from EtOAC-12 fraction by preparative HPLC. (C) Cell viability was examined with isolated compounds. (D) Quantified protein level of MMP-1 in cells pretreated with isolated compounds. Results are expressed as mean \pm SD of triplicate experiments $\left({ }^{*} \mathrm{P}<0.05,{ }^{\# \#} \mathrm{P}<0.01\right.$, $* * P<0.01$ ) 
active one. Three flavone glycosides were isolated from EtOAc-12 fraction by preparative high performance liquid chromatography (Fig. 2A). The compounds were identified as luteolin-8-C-glucoside (lut-8-C) (1), apigenin-8-C-glucoside (api-8-C) (2), and luteolin-7-O-glucoside (lut-7-O) (3) (Fig. 2B).

None of the compounds showed any significant cytotoxicity up to a concentration of $25 \mu \mathrm{M}$ (Fig. 2C). Analysis of MMP-1 secretion by ELISA revealed significant suppression of MMP-1 secretion by all three compounds (Fig. 2D). Specifically, lut-8-C (1) showed the strongest inhibition effect on MMP-1 at $20 \mu \mathrm{M}$. This was comparable to inhibition levels of EGCG at $10 \mu \mathrm{M}$.

Because MAPK signaling and AP-1 have a critical role in ECM maintenance of the dermis, we investigated the effects of three flavone glycosides on UVB-induced activation of MAPKs and AP-1 complex by western blot (Fig. 3A). Lut-8-C (1) and lut-7-O (3) decreased the phosphorylation levels of JNK, ERK, and p38 that were activated up on UVB irradiation (20 $\mathrm{mJ} / \mathrm{cm}^{2}$ ). Api-8-C (2) suppressed on the phosphorylation of ERK, and p38, but had no effect on the phosphorylation of JNK. The phosphorylation of c-Jun and the expression of c-Fos were enhanced by UVB irradiation $\left(20 \mathrm{~mJ} / \mathrm{cm}^{2}\right)$. However, three compounds strongly suppressed their levels (Fig. 3B). Lut-8-C (1) showed the strongest effect at reducing phospho-cJun. Taken together, all three compounds exhibited an inhibitory activity towards MAPKs and AP-1 signaling in the background of UVB exposure.

\section{Antioxidant activity of three flavone glycosides via their $\mathrm{OH}$ groups in UVB-induced $\mathbf{H s 6 8}$ cells}

To estimate antioxidant activity of three compounds, the bond dissociation energies (BDE) of flavonoid $\mathrm{OH}$ groups were calculated (14-16) and DPPH radical scavenging activity were evaluated (Table 1). Lut-8-C (1) had the lowest minimal value of $\mathrm{BDE}$ for $\mathrm{OH}$ bonds $\left(\mathrm{BDE}_{\min }\right)$ for $4^{\prime} \mathrm{OH}(75.50 \mathrm{kcal} / \mathrm{mol})$, and a high number of $\mathrm{OH}$ bonds. Lut-7-O (3) and api-8-C (2) showed the same number of $\mathrm{OH}$ bonds, but the $4^{\prime}-\mathrm{OH}$ value of api-8-C (2) was higher by $8.66 \mathrm{kcal} / \mathrm{mol}$. Concomitant to BDE analysis, api-8-C (2) showed the lowest DPPH radical scavenging activity among three compounds. Altogether, the compounds were found to possess scavenging properties for ROS with several $\mathrm{OH}$ bonds, and lut-8-C (1) showed the strongest effect on antioxidant activity among the three flavone glycosides. In the western blot analysis, lut-8-C (1) and api-8-C (2) had the strong effect on the elevation of antioxidant related proteins, Nrf2 and HO-1 in a dose dependent (Fig. 3C) and time dependent manner (Fig. 3D). Whereas api-8-C (2) only showed significant upregulation of total collagen in Hs68 (Fig. $3 \mathrm{E})$, all flavone glycosides were suppressed ROS production by UVB (Fig. 3F).

\section{DISCUSSION}

Skin aging is a cumulative process induced by genetic, environmental and stress factors and is mainly caused by UV

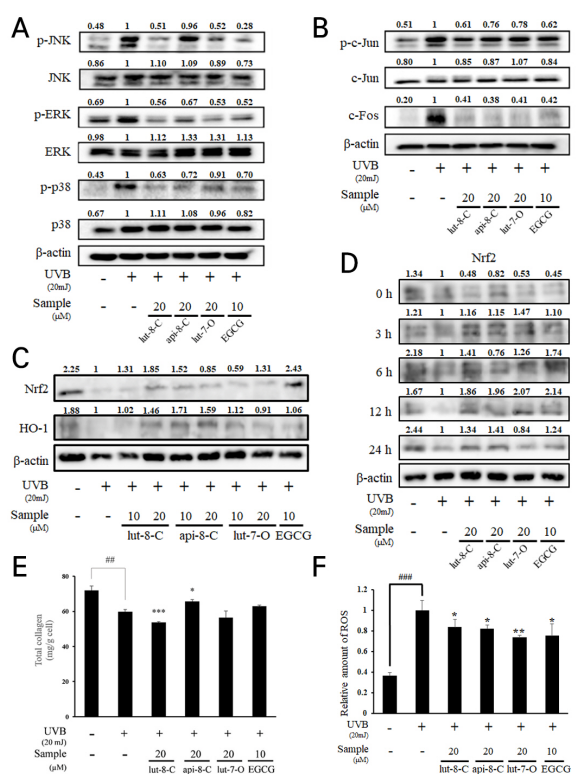

Fig. 3. Effects three flavone glycosides on protein expression of MAPKs, anti-oxidant enzyme and total collagen production in UVB-induced Hs68 cells. (A) MAPKs (JNK, ERK and p38), (B) AP-1 signaling (phosphor-C-Jun and C-Fos) and (C), (D) antioxidant enzymes (Nrf2 and $\mathrm{HO}-1$ ) were determined by western blot analysis. $\beta$-actin was used as an internal control. $(\mathrm{E})$ total collagen production were determined by ELISA assay. (F) ROS level was measured by fluorescence analysis with DCFDA. Results are expressed as mean \pm SD of triplicate experiments $(* P<0.05$, $* * P<0.01,{ }^{\# \#} P<0.01, * * * P<0.001,{ }^{\# \# \# P<0.001)}$.

Table 1. $\mathrm{BDE}$ values for all $\mathrm{OH}$ positions, number of $\mathrm{OH}$ groups, Trolox equivalent antioxidant capacity (TEAC) and EC50 value in three flavones

\begin{tabular}{|c|c|c|c|c|c|c|c|}
\hline \multirow{2}{*}{ Name } & \multicolumn{5}{|c|}{ BDE (kcal/mol) } & \multirow{2}{*}{ TEAC (g TE/g) } & \multirow{2}{*}{$\mathrm{EC}_{50}$ value $(\mathrm{mg} / \mathrm{L})$} \\
\hline & $3^{\prime} \mathrm{OH}$ & $4^{\prime} \mathrm{OH}$ & $5 \mathrm{OH}$ & $7 \mathrm{OH}$ & $\mathrm{nOH}$ & & \\
\hline Luteolin-8-C-glucoside & 86.25 & 75.50 & 90.17 & 89.79 & 4 & $0.628 \pm 0.004^{b}$ & $56.179 \pm 1.277^{\mathrm{b}}$ \\
\hline Apigenin-8-C-glucoside & & 84.78 & 88.40 & 89.31 & 3 & $0.015 \pm 0.007^{c}$ & $4,339.568 \pm 228.643^{\mathrm{a}}$ \\
\hline Luteolin-7-O-glucoside & 80.56 & 76.12 & 88.02 & & 3 & $0.649 \pm 0.005^{\mathrm{a}}$ & $55.902 \pm 1.510^{\mathrm{b}}$ \\
\hline
\end{tabular}


radiation mediated photoaging $(1,3)$. Photoaged skin is accompanied by dryness, wrinkles, laxity, uneven and pigmentation, and increased the risk of skin cancer (17). Therefore, the protection from photoaging by UV radiation is a critical issue for human health. Here, we have demonstrated the protective effects of $H$. japonicus against UVB irradiation and elucidated its mechanisms.

Dermal fibroblasts consist of human dermis and are primary cell types for production of collagens (18). With aging, they show adverse activity of ECM maintenance as well as low production of collagens, suggesting their critical role in skin aging. Under UVB irradiation, Hs68 fibroblasts increased MMP-1 secretion, showing its activity on ECM degradation by UVB induction. However, HJE and its active compounds inhibited MMP-1 secretion from Hs68 cells and modulated AP-1 signaling. In addition, the induction of phosphorylation on MAPK by HJE and its active compounds suggested that HJE exhibits a protective effect in dermal fibroblasts against UVB irradiation, resulting in protection of the skin against UV.

ROS generation induced by UV is the main stimulator of the damage to skin cells $(3,19)$. Therefore, control of ROS level is important for the prevention of skin aging. Luteolin and apigenin, major compounds in the HJE, have been extensively investigated for their diverse biological activities such as the inhibition of oxidative stress through the ERK2/Nrf2 pathway. Because of the different number of $\mathrm{OH}$ groups, the relative order of antioxidant effect was luteolin $>$ apigenin, when analyzed by diphenyl-2-picrylhydrazl (DPPH) method and xanthine/xanthine oxidase system in the skin (18) and TEAC assay (20). Lut-8-C (1) and lut-7-O (3) from HJE showed lower BDE values than api-8-C (2) due to their backbone structure. Furthermore, glycosylation of $\mathrm{C}$ at position 8 in flavone glycosides is known to increase antioxidant capacity because glycosylation of $\mathrm{C}$ at position 8 enhances the stability of the compound by its reduced negative charge (21-23). Therefore, the most stable radical was found in lut-8-C (1) because of its backbone structure (luteolin), C-8 glycosylation and a large number of aromatic hydroxyl groups that is essential for radical scavenging.

However, unlike the expected antioxidant activity based on BDEs, the measurement of antioxidant activity based on the DPPH method showed that lut-7-O (3) had the strongest activity. These data suggest two possibilities: 1) separate activity of HJE distinct from antioxidant activity could participate in the protection of skin cells against UVB, and 2) the DPPH estimation could be less accurate than that of BDE expectation in vitro. The first possibility was demonstrated by collagen estimation, indicating that api-8-C (2) had the best activity on collagen synthesis, while it showed similar effect on the expression of antioxidant enzymes to lut-8-C (1). As for the second possibility, elevated $\mathrm{HO}-1$ and $\mathrm{Nrf} 2$ by lut-8-C (1) demonstrated that antioxidant activity predicted by $\mathrm{BDE}$ calculations is more accurate in cells than DPPH assay, indicating that antioxidant activity of compounds could be estimated differently between cells based on the chemical reaction used. These results suggested that the protective effects of HJE on dermal fibroblasts against UVB arise from the antioxidant properties of the three compounds including lut-8-C (1).

In conclusion, we demonstrated that the flavone glycosides lut-8-C (1), api-8-C (2), and lut-7-O (3) isolated from $H$. japonicus suppressed UVB-induced MMP-1 production in Hs68 cells via decreasing the oxidative stress through JNK, ERK, and p38 MAPKs and AP-1 signaling. Therefore, $H$. japonicus can be used as a constituent of medicines or cosmetics to protect the skin from the effects of UV radiation.

\section{MATERIALS AND METHODS}

\section{Chemical reagents}

Fetal bovine serum (FBS), penicillin and Dulbecco's-modified Eagle's medium (DMEM) were purchased from Hyclone (South Logan, USA). Antibodies for phospho-p44/42 MAPK (ERK1/2 Thr202/Tyr204), ERK1/2, phospho-stress-activated protein kinase (SAPK)/c-Jun (JNK) (Thr183/Tyr185), SAPK/JNK (Thr183/Tyr185) phospho-c-Jun, c-Jun, c-Fos and $\beta$-actin were purchased from Cell Signaling Technology Inc. (Danvers, USA).

\section{Sample preparation}

Aerial parts of $H$. japonicus were purchased from Jirisanman Company (Gokseong-gun, South Korea). The dried aerial parts of $H$. japonicus $(6.0 \mathrm{~kg})$ were repeatedly extracted with $\mathrm{MeOH}$ $(10.0 \mathrm{~L}) 3$ times a day. The extract $(403.6 \mathrm{~g})$ was poured into distilled water and partitioned with Hexane (Hex, $89.0 \mathrm{~g})$, $\mathrm{CH}_{2} \mathrm{Cl}_{2}(15.0 \mathrm{~g})$, Ethyl acetate (EtOAc, $\left.21.3 \mathrm{~g}\right)$, buthanol $(\mathrm{BuOH}, 100.0 \mathrm{~g})$, and $\mathrm{H}_{2} \mathrm{O}$ (178.3 g) fractions successively. Three compounds isolated from the EtOAc fraction were obtained by preparative high performance liquid chromatography. In order to isolate active compounds, EtOAc extract (21.3 g) was subjected to silica gel (Merck, no. 7734) column chromatography and eluted with $\mathrm{CH}_{2} \mathrm{Cl}_{2}-\mathrm{MeOH}(10: 0 \rightarrow$ 10 : 5) monitoring by thin layer chromatography. The compounds were determined based on various spectroscopic methods such as 1D-NMR $\left({ }^{1} \mathrm{H},{ }^{13} \mathrm{C}\right), 2 \mathrm{D}-\mathrm{NMR}$ (gCOSY, gHSQC, gHMBC), IR and mass spectroscopy.

\section{Cell culture and UVB irradiation}

Hs68, human fibroblast cell line (the American Type Culture Collection, Manassas, USA) were cultured in DMEM supplemented with $10 \%$ FBS and 100 units $/ \mathrm{ml}$ penicillin under $5 \% \mathrm{CO}_{2}$ in a humidified atmosphere at $37^{\circ} \mathrm{C}$. Cells were cultured to $80 \%$ confluence, then pretreated with various concentrations of each samples in serum-free medium for $1 \mathrm{~h}$. After washed with fresh phosphate-buffered saline (PBS), cells were irradiated with UVB light $\left(20 \mathrm{~mJ} / \mathrm{cm}^{2}\right)(\mathrm{VL}-6 . \mathrm{LM}$, Vilber Lourmat, Marne-la-Vallee Cedex1, France). UV strength was measured with a Waldman UV meter (model 585100, Waldman, Wheeling, USA). After irradiation, cells were 
incubated in fresh serum-free culture media with various concentration of HJE, HJF, and each compound.

\section{Cell viability}

Cell viability was measured with 3-(4,5-dimethylthiazol-2-yl)2,5-diphenyltetrazolium bromide (MTT) colorimetric assay (Sigma-Aldrich, St. Louis, USA). After UVB irradiation and sample treatments, $0.5 \mathrm{mg} / \mathrm{ml}$ MTT was added to the cells then incubated for $2 \mathrm{~h}$ and the absorbance was measured with a microplate reader at $550 \mathrm{~nm}$ (Bio-Tek Instruments, Winooski, USA).

\section{Enzyme-linked immunosorbent assay}

After UVB irradiation and sample treatments, cell culture medium was collected to measure MMP-1 (human MMP-1 enzyme-linked immunosorbent (ELISA) kit, Abnova, Taipei, Taiwan), and cells were collected with scraper to quantify total collagen (the total collagen ELISA kit (BioVision, Milpitas, CA, USA).

\section{Western blot analysis}

After UVB irradiation and sample treatments, Hs68 cells were lysed in RIPA buffer containing protease inhibitors and protein concentrations of cell lysates were quantified using Bradford protein assay (Bio-Rad Laboratories, Hercules, USA). Equal amounts of proteins $(10 \mu \mathrm{g})$ from each sample were loaded and separated by $10 \%$ sodium dodecyl sulfate-polyacrylamide gel electrophoresis and then transferred to PVDF membranes (Whatman $\mathrm{GmbH}$, Dassel, Germany). Membranes were blocked with $5 \%$ bovine serum albumin (Sigama-Aldrich), and reacted with specific antibodies (Santa Cruz Biotechnology, Dallas, USA). Proteins were detected with enhanced pierce ECL substrate (Thermo Fisher Scientific, Waltham, USA) and visualized using an ChemiDoc ${ }^{\text {TM }}$ XRS + system (Bio-Rad Laboratories, Hercules, USA). Antibodies against $\mathrm{p}^{-} \mathrm{JNK}$, JNK, p-ERK, ERK, p-38, p-p-38, c-fos, p-c-Jun, and c-Jun were purchased from Cell Signaling Technology (Danvers, MA). Antibodies against $\beta$-actin, $\mathrm{HO}-1$, and secondary anti-rabbit and anti-mouse antibodies were purchased from Santa Cruz Biotechnology, Dallas, TX. The antibody against Nrf2 was purchased from Abcam (Cambridge, MA).

\section{Reactive oxygen species (ROS) measurement in UV-B irradiated human fibroblast cell}

HS 68 cells were cultured in 96-well plates $\left(6 \times 10^{3}\right.$ cells/well), and maintained for $24 \mathrm{~h}$. After washing cells with PBS once, the $20 \mu \mathrm{M}$ of lut-C, api-8-C, and lut-7-O, or $10 \mu \mathrm{M}$ of EGCG in serum free media were treated for $1 \mathrm{~h}$. Cells were rinsed with PBS again, and $50 \mu \mathrm{L}$ of PBS was put into each wells. The UVB was irradiated $\left(20 \mathrm{~mJ} / \mathrm{cm}^{2}\right)$ to cells, and remaining PBS was eliminated. For detecting ROS in cells, 20 $\mu \mathrm{M}$ of $2^{\prime}, 7^{\prime}$-dichlorofluoresceindiacetate (DCFDA) in serum free media was added to each well. After $30 \mathrm{~min}$, the fluorescence intensity was measured at $495 \mathrm{~nm}$ for excitation and at $530 \mathrm{~nm}$ for emission.

\section{Calculation of bond dissociation energy}

Bond dissociation energies of lut-8-C (1), api-8-C (2), and lut-7-O (3) were calculated by a quantum chemical calculation method. Structures of three flavone glycosides were retrieved from PubChem database (24). Three-dimensional coordinates for each retrieved molecule were predicted and converted into molecular orbital package software package (MOPAC) input file formats by Open Babel (25). Molecular structures were geometrically optimized and their heat of formation $\left(\Delta \mathrm{H}_{f}\right)$ was calculated by PM7 (26) semi-empirical quantum chemical calculation method using the MOPAC 2016 software (Stewart Computational Chemistry, CO, USA). The bond dissociation energies of $\mathrm{O}-\mathrm{H}$ bond (BDE) were calculated from the following equation:

$$
\mathrm{BDE}=\Delta \mathrm{H}_{f}(\mathrm{AO} \cdot)+\Delta \mathrm{H}_{f}(\mathrm{H})-\Delta \mathrm{H}_{f}(\mathrm{AOH})
$$

Where $\Delta \mathrm{H}_{f}(\mathrm{AO} \cdot), \Delta \mathrm{H}_{f}(\mathrm{H})$, and $\Delta \mathrm{H}_{f}(\mathrm{AOH})$ represent heat of formations of a hydrogen abstracted oxy radical of a phenolic compound, a hydrogen atom and a phenolic compound molecule, respectively.

\section{DPPH radical scavenging activity}

DPPH radical scavenging activities of ut-8-C (1), api-8-C (2), and lut-7-O (3) were analyzed using a method developed by Brand-Williams' group (27).

\section{Statistical analysis}

All data were presented as mean \pm standard deviation (SD) of three determinations. Statistical analyses were performed using the statistical software, GraphPad Prism (GraphPad Software Inc, La Jolla, USA). Group differences were determined by one-way analysis of variance (ANOVA) and Student's t-test followed by Dunnett test.

\section{ACKNOWLEDGEMENTS}

This work was supported by the National Research Council of Science \& Technology (NST) grant by the Korea government (MSIP) (No. CRC-15-01-KIST).

\section{CONFLICTS OF INTEREST}

The authors have no conflicting interests.

\section{REFERENCES}

1. Bosch R, Philips N, Suarez-Perez JA et al (2015) Mechanisms of photoaging and cutaneous photocarcinogenesis, and photoprotective strategies with phytochemicals. Antioxidants (Basel) 4, 248-268

2. Fisher GJ, Wang ZQ, Datta SC et al (1997) Pathophysiology of premature skin aging induced by ultraviolet light. N Engl J Med 337, 1419-1428 
3. Jenkins G (2002) Molecular mechanisms of skin ageing. Mech Ageing Dev 123, 801-810

4. Narayanan DL, Saladi RN and Fox JL (2010) Ultraviolet radiation and skin cancer. Int J Dermatol 49, 978-986

5. Quan T, Qin Z, Xia W et al (2009) Matrix-degrading metalloproteinases in photoaging. J Investig Dermatol Symp Proc 14, 20-24

6. Fisher GJ and Voorhees JJ (1998) Molecular mechanisms of photoaging and its prevention by retinoic acid: ultraviolet irradiation induces MAP kinase signal transduction cascades that induce Ap-1-regulated matrix metalloproteinases that degrade human skin in vivo. J Investig Dermatol Symp Proc 3, 61-68

7. Naya Y and Kotake M (1970) Constituents of Hops. 5. volatile composition of humulus-japonicus sieb et zucc. Bull Chem Soc Jpn 43, 3594-3596

8. Hwang SY, Jung HJ, Jang WS et al (2009) Antiinflammaory effects of the $\mathrm{MeOH}$ extract of Humulus japonicus in vitro. J Korean Med Ophthalmol Otolaryngol Dermatol 22, 71-79

9. Lee YR, Kim KY, Lee SH et al (2012) Antioxidant and antitumor activities of methanolic extracts from Humulus japonicus. Korean J Food \& Nutr 25, 357-361

10. Sung B, Chung JW, Bae HR, Choi JS, Kim CM and Kim ND (2015) Humulus japonicus extract exhibits antioxidative and anti-aging effects via modulation of the AMPK-SIRT1 pathway. Exp Ther Med 9, 1819-1826

11. Yu BC, Yang MC, Lee KH et al (2007) Norsesquiterpene and steroid constituents of Humulus japonicus. Nat Prod Sci 13, 332-336

12. Yu BC, Yang MC, Lee KH et al (2007) Two new phenolic constituents of Humulus japonicus and their cytotoxicity test in vitro. Arch Pharm Res 30, 1471-1475

13. Kim JE, Shin MH and Chung JH (2013) Epigallocatechin-3gallate prevents heat shock-induced MMP-1 expression by inhibiting AP-1 activity in human dermal fibroblasts. Arch Dermatol Res 305, 595-602

14. Amic D and Lucic B (2010) Reliability of bond dissociation enthalpy calculated by the PM6 method and experimental TEAC values in antiradical QSAR of flavonoids. Bioorg Med Chem 18, 28-35

15. Amic D, Stepanic V, Lucic B et al (2013) PM6 study of free radical scavenging mechanisms of flavonoids: why does $\mathrm{O}-\mathrm{H}$ bond dissociation enthalpy effectively represent free radical scavenging activity? J Mol Model 19, 2593-2603

16. Zhang HY, Sun YM and Wang XL (2003) Substituent effects on $\mathrm{O}-\mathrm{H}$ bond dissociation enthalpies and ionization potentials of catechols: a DFT study and its implications in the rational design of phenolic antioxidants and elucidation of structure-activity relationships for flavonoid antioxidants. Chemistry 9, 502-508

17. American Cancer Society, Cancer Facts \& Figures 2019. https://www.cancer.org/content/dam/cancer-org/research/ cancer-facts-and-statistics/annual-cancer-facts-and-figures/2 019/cancer-facts-and-figures-2019.pdf

18. Sim GS, Lee BC, Cho HS et al (2007) Structure activity relationship of antioxidative property of flavonoids and inhibitory effect on matrix metalloproteinase activity in UVA-irradiated human dermal fibroblast. Arch Pharm Res 30, 290-298

19. Rittie L and Fisher GJ (2002) UV-light-induced signal cascades and skin aging. Ageing Res Rev 1, 705-720

20. Benavente-Garcia O, Castillo J, Lorente J et al (2000) Antioxidant activity of phenolics extracted from Olea europaea L. leaves. Food Chem 68, 457-462

21. Perveen S, El-Shafae AM, Al-Taweel A et al (2011) Antioxidant and urease inhibitory C-glycosylflavonoids from Celtis africana. J Asian Nat Prod Res 13, 799-804

22. Praveena R, Sadasivam K, Deepha V et al (2014) Antioxidant potential of orientin: A combined experimental and DFT approach. J Mol Struct 1061, 114-123

23. Praveena R, Sadasivam K, Kumaresan R et al (2013) Experimental and DFT studies on the antioxidant activity of a C-glycoside from Rhynchosia capitata. Spectrochim Acta A Mol Biomol Spectrosc 103, 442-452

24. Wang Y, Xiao J, Suzek TO et al (2009) PubChem: a public information system for analyzing bioactivities of small molecules. Nucleic Acids Res 37, W623-W633

25. O'Boyle NM, Banck M, James CA et al (2011) Open Babel: An open chemical toolbox. J Cheminform 3, 1

26. Stewart JJ (2013) Optimization of parameters for semiempirical methods VI: more modifications to the NDDO approximations and re-optimization of parameters. J Mol Model 19, 1-32

27. Brand-Williams W, Cuvelier ME and Berset C (1995) Use of a free-radical method to evaluate antioxidant activity. Food Sci Technol-Leb 28, 25-30 